\title{
Exercise Fatigue Induce the Oxidative Stress and the Expression of mGluR 4 and mGluR 5 on the Ventrolateral Thalamus in Rats
}

\author{
Meihua $\mathrm{Su}^{1}$, Yong $\mathrm{Mao}^{1}$, Dan $\mathrm{Xu}^{2}$ \\ ${ }^{I}$ School of Physical Education, Jimei University, Xiamen, Fujian, China \\ ${ }^{2}$ College of P. E. and Sports, Beijing Normal University, Beijing, China \\ *Corresponding author.
}

\begin{abstract}
Objective: The motor thalamus plays an important role during exercise. It aims to observe the changes of $m G l u R 4$ and mGluR5 in ventrolateral thalamus of rats induced by repeated exhaustive exercise, and to discuss the relationship of $m G l u R 4, m G l u R 5$ and oxidative damage occured during exhaustive exercise. Methods: There were 48 male wistar rats, which were randomly divided into four groups including $C G, O E G, 24 E G$ and $48 E G$, each group having 12 rats. Meanwhile, immunohistochemistry (IMM) technique was used to investigate the expression of positive cell and integrated optical density (IOD) of metabotropic glutamate receptor 4 (mGluR4) and metabotropic glutamate receptor 5 ( $m$ GluR5), and the impact of exhaustive exercise played on oxidative stress indexes such as malondialdehyde (MDA), glutathione peroxidase (GSH-PX) and superoxide dismutase (SOD) in ventrolateral thalamus of rats were also investigated in different groups. Results: Compared with CG, the expression of mGluR4 and mGluR5 protein of ventrolateral thalamus in OEG and 24EG both significantly increased, and the value of $m G l u R 4$ in $48 E G$ were still higher than control group, however the the value of $m G l u R 5$ in $48 E G$ reduced to the rest level of control group. Meanwhile, the SOD activities of OEG and 24EG group were significantly higher than the control group, and it was the same with GSH content in ventrolateral thalamus of rats. Meanwhile, MDA has been investigated that it increased significantly in OEG and 24EG compared with control group, and the MDA level of 48EG was still significant higher than CG. Moreover, the indexes of muscle injury such as $L D, C K$ and BUN all increased significantly post-exercise immediately and postexercise 24 hours .Conclusion: Exercise fatigue could result in up-regulation of mGluR4 and mGluR5 and increace activity of SOD , GSH-PX and MDA in ventrolateral thalamus of rats, and it also induce the muscle injury by increase the level of $L D, C K$ and BUN in serum, which suggested that ventrolateral thalamus was an important brain rigion to modulate the motor function, and $m G l u R 4$ and $m G l u R 5$ maybe two important receptors prevent from the increase of free radicals and muscle injury induced by exercise fatigue.
\end{abstract}

Keywords: Exhaustive exercise, ventrolateral thalamus, $m G l u R s$, new target supplements

\section{Introduction}

The motor thalamus is connected with many areas of the cerebral cortex, and plays a crucial role in the integration of motor-related information in the basal ganglia, cerebellum and cortex, and the ventrolateral thalamus (VL) is the target nucleus for the surgical treatment of dyskinesia and is the most effective site for the suppression of muscle stiffness ${ }^{[1]}$. Fatigue is an important reason to reduce the working ability of the body. With the further research on the mechanism of central fatigue, the measures to delay central fatigue have become a hot topic. Glutamate is the excitatory neurotransmitter found abundantly in central nervous system, and it is closely related to central fatigue, The metabotropic glutamate receptors play an important role in neurons' excitability when leading the glutamate to nerve fibers, and they constitute potential therapeutic targets for the development of therapies to treat neurodegenerative diseases, currently, mGluR5 may be a novel treatment for Cancer-related Fatigue (CRF) ${ }^{[2]}$. Moreover, metabotropic glutamate receptor 4 (mGluR4) was reported to be related with the anti-inflammatory effect ${ }^{[3]}$.

ISSN: 0010-8189

(C) CONVERTER 2021

www.converter-magazine.info 
As is well known, exhaustive exercise could lead to the increased production of reactive oxygen species (ROS), when the increased free radical surpass the antioxidant defense system occurs, then it will bring about oxidative damage to certain biomolecules in the body ${ }^{[4]}$. The question on how could the reactive oxygen species (ROS) induced by exhaustive exercise affect the expression of mGluR4 and mGluR5 in motor thalamus especially the ventrolateral thalamus (VL) of our central nervous system still remained unclear. Therefore, this study aims to explore the relationship among exercise fatigue, mGluRs and muscle injury indexes such as creatine kinase activity , lactic acid and blood urea nitrogen by observing the change of mGluRs protein in ventrolateral thalamus and reactive oxygen species (ROS) in VL brain tissue after repetitive exhaustive exercise.

\section{Materials and Methodology}

\subsection{Animals and Grouping}

In this study, 48 clean and healthy male wistar rats aging 8 weeks and weighing $262 \pm 10 \mathrm{~g}$, which were provided by Beijing Vital River Laboratory Animal Technology Co., Ltd., and the animal license number was SCXK (Beijing) 2006-0009. The 48 rats were randomly divided into four groups which are control group (CG), exhaustive immediately group (0EG), 24-hour recovery after exercise group (24EG) and 48-hour recovery after exercise group (48EG) with 12 rats in each group. The rats were fed normally and separately, and the food and water were supplied with free access. The room for animal was kept constant with conditions at $(20 \pm 3){ }^{\circ} \mathrm{C}$ and $40 \%-60 \%$ relative humidity, meanwhile it has a cycle of $12 \mathrm{hr}$ dark and $12 \mathrm{hr}$ light. All the experiments were carried out seriously according to the animal ethics committee of Beijing Normal University.

\subsection{Exercise-induced Fatigue Model for Rats}

The rats of control group had been put on the treadmill without doing exercise for 7 days. Meanwhile, rats of exercise groups such as $0 \mathrm{EG}, 24 \mathrm{EG}$ and $48 \mathrm{EG}$ had three days of treadmill training for adaptability for $15 \mathrm{~min}$ every day. Then formal training started from the third day. The exercise groups began to do the training on small animal treadmills at 9:00 a.m every day and continue for 7 days. And the load programs were as following and it had been shown in figure1: (1) 0 incline of the treadmill; (2) Speeds for the three different levels are $8.2 \mathrm{~m} / \mathrm{min}, 15 \mathrm{~m} / \mathrm{min}$ and $20 \mathrm{~m} / \mathrm{min}$. Time duration for the first two levels is 15 minutes and for the third level until exhaustion. The training lasted 7 days. Criteria for exhaustion: animals can not maintain a predetermined speed and tend to stay for a long time on the fixed tailgate even use of sound, light, electrical stimulation can not make them move. Meanwhile, there are symptoms of short breath, abdominal lying on the treadmill and head hanging. Meanwhile, athletic ability, weight and activity status of the animals were observed during the model built. The time to exhaustion was recorded and the average exhaustion time was $153 \pm 15.5 \mathrm{~min}$.

\section{3 laboratory instruments and reagents}

Experimental instruments: SP-801-type animal treadmill, Olympus microscope camera system, Image-Pro Plus6.0 image analysis system (Canada MediaCybernetics Company). Reagents: mGluR4 and mGluR5 resistant and anti-kit, diaminobenzidine (DAB) kit: Beijing Biosynthesis and Bio-Engineering Co., Ltd.; other drugs: paraformaldehyde, disodium hydrogen phosphate, phosphoric acid, sodium dihydrogen sucrose, sodium chloride, chrome alum, gelatin, methanol, 30\% hydrogen peroxide, Triton X-100, ethanol, 95\% ethanol and neutral gum, which are all provided by Beijing Biosynthesis Biotechnology Co., LTD.

2.4 Perfusion, brain-taking, slicing, staining and filming

ISSN: 0010-8189

(C) CONVERTER 2021

www.converter-magazine.info 
After the increasing load treadmill exercise, the control and experimental groups were randomly chosen with 6 rats which were respectively anesthetized with $10 \%$ chloral hydrate. The dose is $3.5 \mathrm{ml} / \mathrm{kg}$. The anesthetized rats were then fixed supine on the operating table. After carefully cutting their abdominal wall and exposing their diaphragm, then we cut their diaphragm and chest wall and pericardium to make the heart exposed. Puncture needles were used to pierce the left ventricle and the aortic root which was quickly fixed with a hemostat. Then we cut a small hole on the right atrial appendage until blood was seen. After that, approximately $200 \mathrm{ml}$ of warm saline was used for perfusion until the liquid flow from the right atrial appendage became clear. And then approximately $200 \mathrm{ml}$ and the pre-cooling $4 \% 4^{\circ} \mathrm{C}$ paraformaldehyde phosphate buffer solution $(0.01 \mathrm{M}, \mathrm{pH}=7.4)$ was used for perfusion for no less than 20 minutes until the liver hardened and whitened. Then the brains of rats were taken away from skull, in addition, they were postfixed in the same fixative solution for 24 hours, then embedded in paraffin. The brain tissue containing the SNR was embedded with paraffin and $5 \mu \mathrm{m}$ thickness of brain sections were prepared for immunohistochemistry. Paraffin sections were routinely dewaxed and hydrated, and then microwave-repaired in $0.01 \mathrm{~m}$ citrate buffer for $20 \mathrm{~min}$. Each section was incubated at $37{ }^{\circ} \mathrm{C}$ for $10 \mathrm{~min}$ with $5010.1 \%$ Triton solution, PBS was rinsed 3 minutes and 3 times, and animal serum was incubated at $37{ }^{\circ} \mathrm{C}$ for $15 \mathrm{~min}$. Then anti-GABA (a) receptor rabbit antibody (1:100) or an anti-GABA (b) receptor rabbit antibody (L: 100) or an anti-mGluR4 rabbit antibody (L: 100) was incubated overnight at $4^{\circ} \mathrm{C}$, rinsed at $0.01 \mathrm{mpbs}(\mathrm{Ph} 7.2)$ for $5 \mathrm{~min}$, and then a second antibody labeled with a working concentration of biotin was added, goat anti-rabbit $\operatorname{IgG}$ labeled with biotin and Rabbit anti-goat IgG labeled with biotin were incubated at room temperature for $20 \mathrm{~min}, 0.01 \mathrm{mpbs}(\mathrm{Ph}$ 7.2) for $3 \mathrm{~min}$, and streptomycin working solution labeled with horseradish enzyme (S-A/HRP) for $20 \mathrm{~min}$ at $37^{\circ} \mathrm{C}$, then it was rinsed with $0.01 \mathrm{~mol} / \mathrm{L}$ PBS $(\mathrm{Ph} 7.2)$ for $3 \mathrm{~min}$; then it was colored with Diaminobenzidine (Dab) for $10-15 \mathrm{Min}$; then it was rinsed with $0.01 \mathrm{~mol} / \mathrm{L} \mathrm{pbs}(\mathrm{Ph} \mathrm{7.2)}$ for $5 \mathrm{~min}$; then the ethanol with different concentration of $95 \%$ I, $95 \%$ II, $100 \%$ I, $100 \%$ II and $100 \%$ III to use to be dehydrated for 1 min respectively; then xylene I and Xylene II were used for transparent for $3 \mathrm{~min}$; the neutral resin were used for sealant; the last, the microscopic observation were used for staining results with $40 \sim 400$ times and photography ${ }^{[5]}$.

\subsection{Estimation of serum CK, BUN and LD}

After the 7 days exhaustion exercise, the control and experimental groups were randomly chosen with 6 rats which were respectively sacrificed at different time. Aliquots of whole blood from eyes of rats were taken immediately for analysis. The blood was centrifuged at $3000 \times \mathrm{g}$ for $10 \mathrm{~min}$ and serum was then aliquoted to cryotubes for various assays. creatine kinase (CK) activity, lactic acid(LD)and blood urea nitrogen (BUN) were estimated by automatic analyser (7170A ,Hitachi) with commercial assay reagents, in order to evaluate the cell membrane injury induced by exercise fatigue.

\subsection{Activity of antioxidant systems}

After the 7 days exhaustion exercise, the control and experimental groups were randomly chosen with 6 rats which were respectively sacrificed at different time. Brain tissue samples were taken away from different groups immediately post-exercise at different recovery time. Ventrolateral thalamus brain tissue was taken and immersed in $0.9 \%$ saline to wash away remaining blood, and then use the filter paper to absorb the moisture. After that, add pre-cooled saline solution at a ratio of 1:9 to the brain tissue to make homogenate. Then the homogenate was centrifuged at $3000 \times \mathrm{g}$ for $15 \mathrm{~min}$ and the supernatant was then dropped to cryotubes for different assays. Then liquid nitrogen was used to flash frozen cryotubes, and the samples were stored at $-80^{\circ} \mathrm{Cfor}$ analysis.The activity of SOD and GSH-PX was detected with a spectrometer according to the method provided by the Nanjing Jiancheng Bioengineering Institute (China). The Superoxide dismutase (SOD) was determined by the method Xanthine oxidase, and the Glutathione Peroxidase (GSH-PX) was determined by the method of DTNB. Lipid peroxidation was determined indirectly by measuring

\section{ISSN: 0010-8189}


malondialdehyde (MDA) which will react with thiobarbituric acid (TBA), then it would be measured at a red species of $532 \mathrm{~nm}^{[5]}$.

\subsection{Statistical analysis}

Five slices were selected from the same two slices in three experimental groups, and five visual fields (400x) were selected in the substantia nigra pars reticular of each slice according to the stereotactic map of the rat brain, the Positive Cell Count and Integrated optical density (IOD) were analyzed by Image-Pro Plus 6.0 true color pathological image analysis system. The data were expressed as mean \pm standard deviation, and the software of SPSS 21.0 was used for statistical analysis. The differences among the groups were tested by One-way Anova. The minimum level of significance was set to be $\mathrm{P}<0.05$.

\section{Data Analysis and Interpretation}

\subsection{Changes of weight in rats during training}

From the table1, after a week of exercise in accordance with the fatigue model, the rats in the control group were raised normally, moved freely, and gained normal weight. However, most of the rats in the exhaustion groups showed malaise and unshiny hair, reduced foraging and activity. Also, it showed significant decline in body weight in three exhaustion groups compared with control group $(\mathrm{P}<0.05)$.

\subsection{IOD of mGluR4 and mGluR5 post exercise fatigue}

The effects of exhaustive exercise on mGluR4 and mGluR5 protein IOD were shown in Figure 2, from which we can see that, compared to the control group, mGluR4 protein IOD in ventrolateral thalamus of 0EG, 24EG and 48EG after repeated exhaustive exercise significantly raised $(\mathrm{P}<0.05, \mathrm{P}<0.001$ and $\mathrm{P}<0.05$ respectively; $\mathrm{n}=6$ ), furthermore, the value of mGluR4 IOD in 24EG was significant higher than $0 \mathrm{EG}(\mathrm{P}<0.05)$, and the value of mGluR4 IOD in 48EG was the same with control group $(\mathrm{P}>0.05)$. Meanwhile the IOD of mGluR5 increased significantly in $0 \mathrm{EG}$ and 24EG $(\mathrm{P}<0.001$ and $\mathrm{P}<0.001$ respectively; $\mathrm{n}=6)$, and the IOD of $\mathrm{mGluR5}$ of $48 \mathrm{EG}$ remained as the almost same level as that of $\mathrm{CG}(\mathrm{P}>0.05 ; \mathrm{n}=6)$, the data of mGluR5 IOD in Figure 2 also presented that there was no significance between $0 \mathrm{EG}$ and 24EG $(\mathrm{P}>0.05 ; \mathrm{n}=6)$, however, IOD of mGluR5 of 48EG reduced significantly compared with $0 \mathrm{EG}(\mathrm{P}<0.01 ; n=6)$. In summary, the IOD of mGluR4 increased obviously and it did not return to the rest level of control group. However, the IOD of mGluR5 reduced to quiet level after 48 hours.

\subsection{Effects of exhaustive exercise on mGluR4 and mGluR5 positive cells}

The effects of exhaustive exercise have on mGluR4 and mGluR5 positive cells in ventrolateral thalamus were seen in Figure3, from which it could be concluded that compared with CG, the expression of mGluR4 in 0EG, 24EG and 48EG all significantly increased $(\mathrm{P}<0.05, \mathrm{P}<0.001$ and $\mathrm{P}<0.05$ respectively; $\mathrm{n}=6)$, and the positive cells of mGluR4 in 24EG increased significantly compared with $0 \mathrm{EG}$, moreover, there was no difference between 0EG and 48EG of mGluR4 positive cells. At the same time, it also showed that the positive cells of mGluR5 in 0EG, 24EG raised significantly compared with $\mathrm{CG}(\mathrm{P}<0.001$ and $\mathrm{P}<0.05)$, however, the positive cells of mGluR5 in 48EG did not have obvious significance compared with $\mathrm{CG}(\mathrm{P}>0.05 ; \mathrm{n}=6)$. Moreover, the positive cells of mGluR5 in 24EG did not change obviously compared with 0EG $(\mathrm{P}>0.05)$, and its value in 48 EG reduced significantly compared with 0EG. Totally, the positive cells of mGluR5 after exhaustive exercise displayed that its value increased significantly, and then it reduced to quiet level post-exercise 48 hours.

3.4 Measurement of SOD, GSH-PX and MDA

ISSN: 0010-8189

(C) CONVERTER 2021

www.converter-magazine.info 
From the table2, it showed that the SOD activities in ventrolateral thalamus brain tissue of rats were largely altered in all groups. The SOD activities of $0 \mathrm{EG}$ and 24EG group were significantly lower than the control group (both $\mathrm{P}<0.001$ ), but there was no significance between $\mathrm{CG}$ and 48EG. The content of GSH-PX in ventrolateral thalamus of rats reduced significantly of $0 \mathrm{EG}$ and 24EG compared with control group $(\mathrm{P}<0.001$ and $\mathrm{P}<0.05$ respectively), and it did not displayed significant difference between 48EG and CG. However, the content of GSH-PX in 24EG was significant higher than the content of $0 \mathrm{EG}(\mathrm{P}<0.05)$, and there was no difference between 24EG and 48EG.The level of MDA in ventrolateral thalamus of $0 \mathrm{EG}$ and 24EG increased significantly compared with $\mathrm{CG}(\mathrm{P}<0.001$ and $\mathrm{P}<0.01$ respectively), and the MDA level of 48EG was still significant higher than CG $(\mathrm{P}<0.05)$. All in all, the indexes of antioxidant system such as SOD and GSH-PX decreased immediately post exercise, and then returned to the quiet level of pre-exercise. Lipid peroxidation was expressed by the value of MDA level, and its content increased significantly in all group, it means the level of MDA in serum did not dropped to the rest of control group.

\subsection{Concentration of LD, CK and BUN in serum}

From the table3, it showed that the concentration of LD in serum significantly changed post exhaustive exercise. The value of LD in 0EG and 24EG group significantly increased compared with control group (both $\mathrm{P}<0.001$ ), and its concentration returned to rest level of control group. More over, the concentration of LD in 24EG was lower than $0 \mathrm{EG}(\mathrm{P}<0.05)$. The activities of $\mathrm{CK}$ increased obviously in $0 \mathrm{EG}$ and 24EG group (both $\mathrm{P}<0.001)$, and its value was still higher than the control group $(\mathrm{P}<0.05)$. Meanwhile, the level of BUN increased significantly in $0 \mathrm{EG}$ and 24EG group $((\mathrm{P}<0.001$ and $\mathrm{P}<0.01$ respectively), and value of BUN returned to the rest level of control group. So the indexes of muscle injury such as LD, CK and BUN all increased significantly post-exercise immediately and post-exercise 24 hours, and then the level of LD and BUN returned to the rest value, however, the activities of CK ware still higher than the rest level.

Table 1 Changes of weight in rats during training $(\bar{x} \pm \mathrm{s}, \mathrm{n}=12)$

\begin{tabular}{lllll}
\hline Time & CG & OEG & $24 \mathrm{EG}$ & $48 \mathrm{EG}$ \\
\hline Pre-exercise & $250.14 \pm 6.32$ & $249.93 \pm 6.84$ & $250.45 \pm 6.42$ & $251.31 \pm 6.72$ \\
Day-1 & $251.57 \pm 7.84$ & $251.78 \pm 7.27$ & $251.76 \pm 5.97$ & $250.78 \pm 7.84$ \\
Day-2 & $253.14 \pm 8.24$ & $252.63 \pm 6.31$ & $251.67 \pm 6.72$ & $251.93 \pm 7.21$ \\
Day-3 & $255.45 \pm 7.36$ & $253.18 \pm 6.25^{*}$ & $252.69 \pm 7.32^{*}$ & $253.27 \pm 6.31^{*}$ \\
Day-4 & $258.72 \pm 8.04$ & $250.43 \pm 4.23^{* *}$ & $248.73 \pm 5.88^{* *}$ & $251.36 \pm 6.25^{* *}$ \\
Day-5 & $261.78 \pm 7.53$ & $248.57 \pm 7.54^{* *}$ & $249.42 \pm 6.28^{* *}$ & $249.49 \pm 8.75^{* *}$ \\
Day-6 & $264.57 \pm 6.72$ & $248.02 \pm 6.27^{* *}$ & $247.75 \pm 6.87^{* *}$ & $248.65 \pm 7.69^{* *}$ \\
Day-7 & $267.48 \pm 6.91$ & $246.27 \pm 7.59^{* *}$ & $247.24 \pm 5.93^{* *}$ & $246.32 \pm 6.68^{* *}$ \\
\hline
\end{tabular}

Compared with Control group, ${ }^{*} P<0.05,{ }^{* *} P<0.01$.

ISSN: 0010-8189

(C) CONVERTER 2021

www.converter-magazine.info 
Volume 2021, No. 5

Table 2 The changing of SOD, GSH-PX and MDA on ventrolateral thalamus tissue in different time groups

\begin{tabular}{llll}
\hline Group & SOD (U/mgprot) & GSH-PX(U/mgprot) & MDA(nmol/ mgprot) \\
\hline CG & $184.33 \pm 7.47$ & $268.14 \pm 7.20$ & $7.23 \pm 0.73$ \\
OEG & $142.50 \pm 13.26^{* * *}$ & $182.42 \pm 12.59 * * *$ & $13.33 \pm 1.24 * * *$ \\
24EG & $154.66 \pm 7.15^{* * *}$ & $243.71 \pm 13.58^{* *}$ & $10.41 \pm 1.57 * * \#$ \\
48EG & $186.50 \pm 8.64^{\# \# \#}$ & $273.57 \pm 9.38^{\# \# \#}$ & $9.55 \pm 1.31 *^{\# \#}$
\end{tabular}

All the data was expressed by $\mathrm{x} \pm \mathrm{s}$, compared with $\mathrm{CG}, * \mathrm{P}<0.05, * * \mathrm{P}<0.01, * * * \mathrm{P}<0.001$; Compared with $\mathrm{EG}$, ${ }^{\#} \mathrm{P}<0.05,{ }^{\# \#} \mathrm{P}<0.01,{ }^{\# \# \#} \mathrm{P}<0.001$.

Table 3 The value of LD, CK and BUN in SERUM OF different groups

\begin{tabular}{llll}
\hline Group & LD $(\mathrm{mmol} / \mathrm{L})$ & CK $(\mathrm{U} / \mathrm{L})$ & BUN $(\mathrm{mmol} / \mathrm{L})$ \\
\hline CG & $5.64 \pm 0.56$ & $732.43 \pm 132.61$ & $5.01 \pm 0.52$ \\
OEG & $9.56 \pm 0.59^{* * *}$ & $1455.36 \pm 173.78 * * *$ & $8.47 \pm 0.58 * * *$ \\
24EG & $8.66 \pm 0.65^{* * * \#}$ & $1195.40 \pm 126.42^{* * * * \#}$ & $6.28 \pm 0.56^{* * \# \#}$ \\
$48 \mathrm{EG}$ & $6.69 \pm 0.94^{\# \#}$ & $927.35 \pm 111.62^{* \# \#}$ & $5.28 \pm 0.93^{\# \#}$ \\
\hline
\end{tabular}

All the data was expressed by $\mathrm{x} \pm \mathrm{s}$, compared with $\mathrm{CG}, * \mathrm{P}<0.05, * * \mathrm{P}<0.01, * * * \mathrm{P}<0.001$; Compared with $\mathrm{EG}$, ${ }^{\#} \mathrm{P}<0.05,{ }^{\# \#} \mathrm{P}<0.01,{ }^{\# \# \#} \mathrm{P}<0.001$.

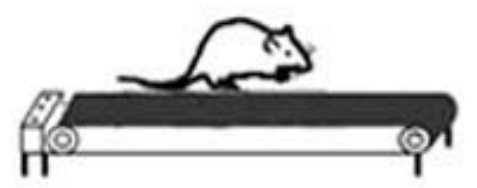

\section{rest exercise exhaustive \\ recovery}

\begin{tabular}{|c|c:c:c|c|}
\hline $30 \mathrm{~min}$ & $\begin{array}{c}\text { I: } 8.2 \mathrm{~m} / \mathrm{min} \\
15 \mathrm{~min}\end{array}$ & $\begin{array}{c}\text { II: } 15 \mathrm{~m} / \mathrm{min} \\
15 \mathrm{~min}\end{array}$ & $\begin{array}{c}\text { III: } 20 \mathrm{~m} / \mathrm{min} \\
\text { until exhaustion }\end{array}$ & $90 \mathrm{~min}$ \\
\hline
\end{tabular}

Figure 1 Exercise Fatigue Model for Rats

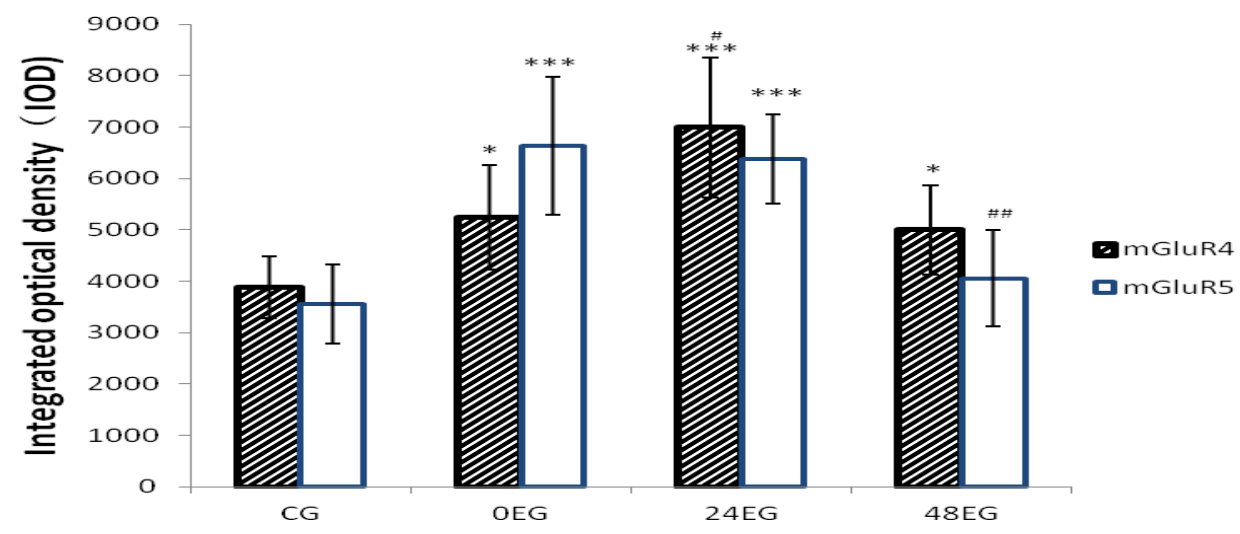

Figure 2 Integrated option density of mGluR4 and mGluR5 expression in ventrolateral thalamus of rats post exhaustive exercise $(\bar{x} \pm s, n=6)$

ISSN: 0010-8189

(C) CONVERTER 2021

www.converter-magazine.info 


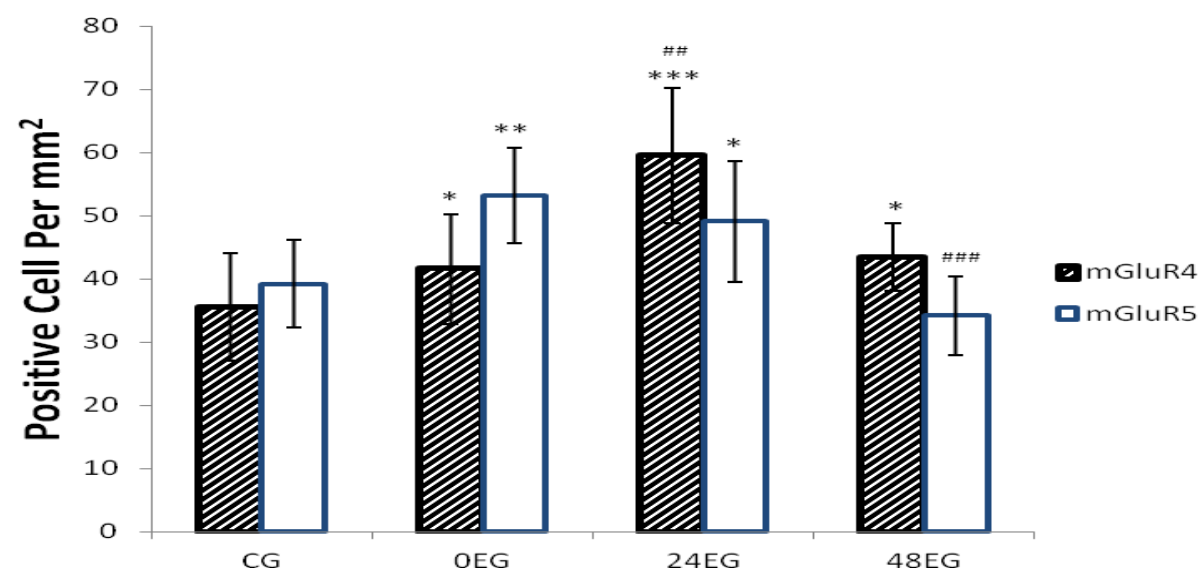

Figure 3 Positive cell number of $m G$ GuR4 and $m G$ GluR5 expression in ventrolateral thalamus of rats after exhaustive exercise $(\bar{x} \pm s, n=6)$

All the data was expressed by $\mathrm{x} \pm \mathrm{s}$, compared with $\mathrm{CG}, * \mathrm{P}<0.05, * * \mathrm{P}<0.01, * * * \mathrm{P}<0.001$; Compared with $\mathrm{EG}$, ${ }^{\#} \mathrm{P}<0.05,{ }^{\# \#} \mathrm{P}<0.01,{ }^{\# \# \#} \mathrm{P}<0.001$.

\section{Discussion}

In the central nervous system, the thalamus is a subcortical sensory center next to the sensory center of the brain, which is involved in sensory production and integration, and it is also involved in maintaining the body's state of arousal ${ }^{[1]}$. Exercise fatigue usually happened during the exhaustive and intensive exercise which would reduce the work power, endurance, velocity, motor coordination and body reaction, moreover, it may lead to the occurrence of injury in some severe cases. As a sensitive index to evaluate the degree of muscle hypoxic fatigue after high intensity or exhaustive exercise, the increase of lactic acid level will further reduce the $\mathrm{PH}$ value and cause physiological side effects ${ }^{[5]}$. Urea is the result of protein metabolism, which causes a significant increase of nitrogen level in peripheral blood and is an important index related to protein decomposition, dehydration and stress, thus it will reduce human endurance and result in fatigue.Intense exercise may cause damage to muscle cells, which release ck into the blood, therefore, the activity of CK in the blood is often assessed as a sign of muscle damage caused by exercise fatigue ${ }^{[6]}$. The rats exercise fatigue model was built up by repeated exhaustive exercise for 7days, and the data in our study presented that the indexes of muscle injury such as LD, CK and BUN all increased significantly in 0EG and 24EG, and then the level of LD and BUN returned to the rest value, however, the activities of CK ware still higher than the rest level. From our data, it suggested that the repeated exhaustive exercise could lead to fatigue and muscle damage until more than 48 hours.

Glutamate is the largest in number of the excitatory neurotransmitters in the central nervous system. Glutamate excitotoxicity was mediated by its receptors mainly including ionotropic receptors (iGluRs) and metabotropic receptors (mGluRs) ${ }^{[2-3]}$.In recent years, glutamate receptor has received considerable attention in the neurological disorder, which is one of the important pathological markers of central nervous system disease, and the up-regulation of mGluR4 expression is closely related to the occurrence and development of depression, which may be one of the causes of depression ${ }^{[3]}$. Metabotropic glutmate receptor 5(mGluR5), which regulates excitatory glutamate transmitters and postsynaptic signals, has attracted much attention in the field of neurobiology as a target of a variety of neurological disorder drugs ${ }^{[2]}$. The study of the effects of

ISSN: 0010-8189

(C) CONVERTER 2021

www.converter-magazine.info 
exhaustive exercise on mGluR4 and mGluR5 expression in ventrolateral thalamus (VL) remained unclear, although recent study implicate the expression of GRM4 was higher in Osteosarcoma than that of GRM1 and GRM5, and the abnormal expression of GRM4 was closely related to the stage and metastasis of Osteosarcoma $^{[7]}$.

The experimental results showed that the expression of mGluR4 and mGluR5 protein of ventrolateral thalamus in 0EG and 24EG both significantly increased, which indicated exhaustive exercise can lead to increasing mGluR4 and mGluR5 expression in ventrolateral thalamus. Increase of neuronal excitability of 0EG and 24EG may be the result of the interaction of glutamate and its receptor mGluR4 and mGluR5, and the expression of mGluR4 and mGluR5 in ventrolateral thalamus neurons may mediate glutamate neurotoxicity. Studies reported ${ }^{[7]}$ that activation of mGluRs receptors could cause neurons excited, which increased neuronal death of glutamate excitotoxicity, and that maybe one of the causes of exercise-induced fatigue.

Reactive Oxygen Species (ROS) are a group of chemical property molecules or ions with high oxidative activity, and It refers to all oxygen containing free radicals in the cell, such as superoxide anion, oxygen free radicals, hydroxyl, peroxide, etc ${ }^{[4]}$. Free radical is essentially an oxidizing molecule or atom with at least one unpaired electron in the outer orbital, moreover, free radicals are formed in the course of normal cellular metabolism, which uses oxygen to generate energy. If free radicals are not quickly neutralized by antioxidants, they can harm the body and cause some diseases ${ }^{[5-6]}$.Superoxide dismutase (SOD) is an important antioxidant enzyme in living organisms, which is the first substance in the body to eliminate free radicals, furthermore, it can resist and block the damage caused by oxygen free radicals and lipid perioxidation, and again it can repair the damaged cells in time and restores cellular damage caused by free radicals ${ }^{[5-6]}$. Malondialdehyde(MDA) is one of the most important products of membrane lipid Peroxidation, which can cause cross-linking polymerization of protein, nucleic acid and other macromolecules, and it has cytotoxicity. The content of MDA is a common index, through which the degree of membrane lipid peroxidation can be known and the damage degree of membrane system and the resistance of organism can be indirectly measured ${ }^{[5-6]}$.

Our study indicated that the SOD activities of 0EG and 24EG group were significantly lower than the control group, and it was the same with GSH-PX in ventrolateral thalamus brain tissue of rats. Meanwhile, malondialdehyde (MDA) is a marker of lipid oxidation, which has been investigated that it increased significantly in 0EG and 24EG compared with control group. And the MDA level of 48EG was still significant higher than CG. So it suggested that the antioxidant enzyme such as SOD and GSH-PX significantly decreased in ventrolateral thalamus brain tissue after exhaustive exercise. The level of SOD and GSH-PX detected in our study were the same with the report that the blood levels of superoxide dismutase (SOD), GPX and serum levels of TAC significantly decreased after intense physical activity ${ }^{[5]}$, meanwhile the lipid peroxidation significantly increased after acute exercise ${ }^{[5]}$.

It also has been proved that exhaustive exercise can cause localized ischemia in rat brains and then a series of pathophysiological responses ${ }^{[8]}$.In our experiment, mGluR4 and mGluR5 protein content in ventrolateral thalamus (VL) of the rat significantly increased in $0 \mathrm{EG}$ and $24 \mathrm{EG}$, which may be due to the fact that rats cannot adapt to the stress stimulus at the beginning of acute exercise, since the free radicals lipid perioxidation and it would started our anti-oxidant system like the enzyme of SOD and GSH-PX in our body to protect from oxidative damage. And therefore focal ischemia appeared which led to the decline in ATP content in the ventrolateral thalamus. Simultaneously, large amount of ATP and oxygen was consumed, which caused relative shortage of ATP and oxygen supply in brain. Study found that acute cerebral ischemia could cause metabotropic glutamate receptors mRNA expression increases, suggesting that mGluRs was involved in focal cerebral ischemic injury and probably contributed to the development of ischemic

ISSN: 0010-8189

(C) CONVERTER 2021

www.converter-magazine.info 
cerebrovascular disease ${ }^{[8]}$, meanwhile, treadmill pre-training has a significant neuroprotective function against ischemia/reperfusion injury, by suppressing glutamate release resulting from cerebral ischemia, and this effect may be mediated by down regulation of metabotropic glutamate receptors ${ }^{[9]}$. It had similarities with the results of our study showing out that mGluR4 and mGluR5 expression was increasing due to exercise-induced ischemia and hypoxia. Therefore, it suggests that the expression mGluR4 and mGluR5 in ventrolateral thalamus (VL) may be the other important receptors related with the production of exercise fatigue induced by oxidative damage especially mediated by free radicals and lipid perioxidation in the central nervous system.

\section{Conclusion}

Repeated exhaustive exercise could lead to exercise fatigue by increasing the level of the indexes of muscle injury such as LD, CK and BUN until post-exercise 24 hours, moreover, it results in up-regulation of expression of mGluR4 and mGluR5 in ventrolateral thalamus of rats at different recovery time. Meanwhile, levels of superoxide dismutase (SOD) and the Glutathione Peroxidase(GPX) significantly reduced postexercise 24 hours, and lipid peroxidation significantly increased until more than 48 hours. It suggested that ventrolateral thalamus is an important brain region to control exercise, which modulate the up-regulation of metabotropic glutamate receptors such as mGluR4 and mGluR5 both related with the incre ase of free radicals induced by exercise fatigue. It suggested that the mGluR4 and mGluR5 would be new target supplements to prevent from exercise fatigue. The limitation of our study was that we did not have more samples to get the data due to the precious animals. Furthermore, we need to do experiment to investigate the different effect of the other subtypes of metabotropic glutamate receptors on ventrolateral thalamus.

\section{Acknowledgment}

This work was supported by Fujian Province Natural Science Fund of Grant No.2019J01754 and Fujian Province Science Found of the Grant No.FJ2018X008 from China, also it is supported by the cultivation grant(No.C111403) from Jimei University and the grant(No.JAS20137) from the Education Department of Fujian Province.

\section{References}

[1] S. Xing, J. Zhang, C. Dang, et al., "Cerebrolysin reduces amylord- $\beta$ deposits, apoptosis and autophagy in the thalamus and improves functional recovery after cortical in farction," J Neurol Sci, vol. 337, no. 1/2, pp. 104-111., 2014.

[2] Feng Li Rebekah, Fernández-Martínez Juan Luis, Zaal Kristien J.M., et al., "mGluR5 mediates postradiotherapy fatigue development in cancer patients," vol. 8,no. 1, pp. 110-121, 2018.

[3] G. Abulwerdi, B. A. Stoica, D. J. Loane, et al., "Putative mGluR4 positive allosteric modulators activate Gi-independent anti-inflammatory mechanisms in microglia," Neurochemistry International,vol. 138, pp. 104770, 2020.

[4] P. Moller, S. Loft, C. Lundby, et al., "Acute hypoxia and hypoxic exercise induce DNA strand breaks and oxidative DNA damage in humans," Faseb J. vol. 15, no. 7, pp. 1181-1186, 2001.

[5] M.h. Su, D. xu, J.m. Chen, "The Effects of Exercise Fatigue on mGluR1 expression and oxidative stress in striatum of rat," ACTA MEDICA MEDITERRANEA, vol. 36, no. 5, pp. 3095-3100, 2020.

[6] S. Zhu, W. Yang, Y. Lin, et al., "Antioxidant and anti-fatigue activities of selenium-enriched peptides isolated from Cardamine violifolia protein hydrolysate," Journal of Functional Foods, vol. 79, pp. 104412, 2021.

[7] W. Yang, MAOLIN H, JINMIN Z, et al., "High expression of metabotropic glutamate receptor 4:correlation with clinicopathologic characteristics and prognosis of osteosarcoma," J Cancer Res Clin Oncol, vol. 140, no. 3, pp. 419-426, 2014.

ISSN: 0010-8189

C CONVERTER 2021

www.converter-magazine.info 
Volume 2021, No. 5

[8] M.S. Yong, S.G. Kim, S.H. Cheon, "Effects of skilled reach training with affected forelimb and treadmill exercise on the expression of neurotrophic factor following ischemia-induced brain injury in rats," J Phys Ther Sci,vol. 29, no. 4, pp. 647-650, 2017.

[9] J. Jia, Y.S. Hu, Y. Wu, "Treadmill pre-training suppresses the release of glutamate resulting from cerebral ischemia in rats," Exp Brain Res. vol. 204, no. 2, pp. 173-179, 2010. 